\title{
Dithiothreitol revisited in red cells: A new head for an old hat
}

\author{
J.P. Lopes de Almeida* and C. Saldanha \\ Institute of Biochemistry, Institute of Molecular Medicine, University of Lisbon Medical School, \\ Lisbon, Portugal
}

\begin{abstract}
In the present article the authors make an approach over the applications of dithiothreitol (DTT) in its different clinicallaboratory, potential and up-to-date sources. Dithiothreitol is a chemical reagent with a wide actuation spectrum not only from a laboratorial view but also from a therapeutic standpoint, more clinical and practical. DTT (i) is frequently used in a variety of experiences that involve proteins or peptides, protecting sulfhydryl groups from oxidation and reducing disulfide bonds between cysteines; (ii) is also used in the study of disulfide exchange reactions of protein disulfides; (iii) is able to keep glutathione in the reduced state; (iv) acts as an "antidote" enabling the activity of detoxification systems; (v) participates in cellular mechanisms such as vesiculation, cell morphology, signal transduction pathways (hormone- 'like' role), etc.; (vi) can be used in the treatment approach of diseases like cystinosis or medical conditions resulting from ion or metal toxicity. In erythrocytes, there's literature pointing that DTT may trigger changes on the normal discoid shape following metabolic depletion, and additionally modulate the exovesiculation kinetics as demonstrated by us. The present article dissects in detail recent findings in our Unit concerning the DTT influence on human erythrocytes.
\end{abstract}

Keywords: Dithiothreitol, erythrocyte, redox status, thiols

\section{Introduction}

1,4-Dithiothreitol (DTT) is the common name for the chemical compound known as Cleland's Reagent, a small-molecule redox reagent featured to be a highly alcohol- and water-soluble solid with little odor and poor tendency to be air-oxidized $[11,30]$. Discovered in 1949 , this synthetic reagent is able to completely reduce disulfides, protect sulfhydryl groups $(-\mathrm{SH})$ from oxidation and preserve monothiols in the reduced state. Thence, DTT plays a key-role as the golden-standard protective reagent for -SH groups, which seems to be critical for the maintenance of biological functions in numerous macromolecules $[1,11,30]$.

DTT applications on humans can be divided into thiol and non-thiol effects, the spectrum of which covers both laboratorial and medical fields. The vastest actuation group encompasses the thiol effect, since the thiol-dissulfide interchange is essential for a wide range of biological systems $[1,14]$ and it is concerned with DTT's redox properties, i.e. the ability to reduce disulfide bonds between cysteines and to work out as a -SH donor. Non-thiol effects regard little interactions in the absence of cysteine (Cys) residues, such as the inhibition of protein-carbohydrate binding [29].

${ }^{*}$ Corresponding author: J.P. Lopes de Almeida, Instituto de Bioquímica, Instituto de Medicina Molecular, Faculdade de Medicina de Lisboa, Edifício Egas Moniz, Av. Prof. Egas Moniz, 1649028 Lisboa, Portugal. Tel.: +351 91 8985450; Fax: +351 21 7999477; E-mail: jpedro.gla@gmail.com. 


\section{Chemical approach}

Dithiothreitol is a peculiarly potent reducing agent, owing to its conformational propensity to form a six-membered ring with disulfide bonds. The typical reduction of a disulfide bond encompasses two sequential thiol-disulfide exchange reactions. The intermediate mixed-disulfide state is generally unstable because the second DTT thiol sustains a high propensity to close the ring yielding oxidized DTT and a reduced disulfide bond behind. Characteristically, the reducing power of DTT is limited to $\mathrm{pH}>7[11,30]$.

On the one hand, DTT is usually used to reduce disulfide bonds and prevent intra- and intermolecular disulfide bonds from yielding between cysteine residues of proteins. It is yet important to note that solvent-inaccessible cannot be reduced by this reagent. DTT can also be used as an oxidizing agent and in opposition to what happens with glutathione no mixed-disulfide species are populated [30].

On the other hand, DTT is commonly used as a deprotecting agent for thiolated DNA, which has a strong tendency to form dimers in solution in the presence of oxygen. This dimerization process importantly decreases the efficiency of subsequent coupling reactions such as DNA immobilization on gold in biosensors. DTT typically reacts with a DNA solution, and afterwards is removed by filtration or chromatography $[1,11,30]$.

\section{What we do know after decades of DTT usage}

In line with the literature behind there's an increasing body of evidence of ground-breaking dithiothreitol applications in the clinical setting, due to its little toxicity. A brief review of potential applications follows herein:

(i) The protection of DNA from oxidation by inhibiting the production of oxygen free radicals, extremely useful in procedures of DNA transformation [19].

(ii) Treatment option for patients suffering from cystinosis, since DTT efficiently decreases the intracellular levels of cysteine in human leucocytes, especially in neutrophils where its stockpile is larger [17].

(iii) The protection of numerous cells from oxidative stress damage, by acting as an antidote and thus keeping the endogenous antioxidant glutathione in the reduced state [29].

(iv) The reduction of several toxic drugs, such as acetaminophen, to their nontoxic parental compounds. Independently of the glutathione levels, DTT is known to revert drug-induced hepatotoxicity by interacting with $-\mathrm{SH}$ groups of $\mathrm{Ca}^{2+}$-ATP translocases [23, 37].

(v) The protection of erythrocytes against organometal-induced hemolysis, specifically by organolead compounds, which is lipophilicity-dependent. DTT triggers an anti-hemolytic effect by inhibiting the interaction between those toxic agents and cellular membranes [3,21].

(vi) The enhancement of metal ions (mercury and copper) toxicity, characterized by high affinity to thiol groups and glutathione depletion as the main causes for cell damage, whereas no affection on glutathione levels has been detected after DTT treatment [20].

(vii) Modulation of the activity of surface markers in white blood cells via down-regulation of its expression, which may thereby prompt an immune depression condition [40].

(viii) The formation of DTT complexes with metals (e.g. zinc-DTT) avoiding its enzyme inhibition effect on $\mathrm{Na}^{+}-\mathrm{K}^{+}$ATPases, which may then be responsible for severe neurological disorders [28]. 
(ix) The formation, by DTT, of novel disulfide bonds between the five subunits that constitute the acetylcholine nicotinic receptor, which blocks its normal assembly and hinders the number of receptors within the membrane.

(x) The induction by DTT of numerous cell adhesion molecules, thereby enhancing the adhesive ability of neutrophils, natural-killer cells and platelets [15, 36]. In the hemorheology setting, DTT further stimulates platelet aggregation [41].

(xi) The hormone-like effect of DTT, yet found on adipocytes, which is based on the interaction with insulin tyrosine-kinase receptors (molecules that also contain sulfhydryl groups). The reducing agent mimetizes the insulin metabolic effects, therefore activating glucose uptake and prompting an anti-lipolytic effect $[18,22]$.

(xii) The recommendation of DTT for routine usage in blood banking, with the purpose of inactivating IgM antibodies and as well as a test to differentiate IgM from IgG [27].

(xiii) The DTT-dependent redox signalling in oxygen sensing by natal and postnatal vessels: inhibition of potassium currents in the pulmonary artery, thereby triggering vasoconstriction by increased cytosolic calcium, with the opposite effect being verified within the ductus arterious [4].

\section{Modulation of erythrocyte properties by DTT}

The modulation of erythrocyte physiological events by dithiothreitol is becoming increasingly documented $[34,38,39]$ and ongoing studies from our unit are disclosing its thiol-reducing property to be the major role in erythrocyte function.

Reducing agents regulate cellular shape which does not occur through cytoskeleton proteins phosphorylation or phosphate turnover, but via phosphorylation of lipids bound to the inner membrane yielding phosphatidic acid and PIP3. Upon erythrocyte crenation, a major consequence of metabolic depletion, a trigger of normal discoid shape onto echinocyte is observed, which can be reverted with substrate supplementation. In DTT-treated cells, this morphology deformation goes further until the final form is a stomatocyte. This counter-regulation is the result of exaggerated stimulation of the plasma membrane aminophospholipid translocator by DTT [38, 39].

Current studies from our laboratory have revealed dithiothreitol effects on erythrocyte exovesiculation [34], hitherto a poorly understood mechanism. Exovesiculation is known to occur during red cell differentiation, apoptosis and during the process of blood samples storage, and it plays an important role as a homeostasis effective modulator. The release of exovesicles is strongly modulated by $\mathrm{pH}$, temperature and ATP depletion. Those microvesicles are endowed with the membrane-bound acetylcholinesterase, an enzyme documented to embody the erythrocyte integrity. Our research group has recently developed an experimental design to study erythrocyte membrane exovesiculation in the presence of the fluorescent membrane probes DPH, TMA-DPH, and $\mathrm{C}_{17}-\mathrm{HC}$ [33].

\subsection{DTT and erythrocyte rheology}

Over the last years, we have published a few studies exploring the non-neuronal cholinergic system in human erythrocytes [6-10, 12, 13, 26, 31, 32, 35]. There is growing knowledge about the association between hemorheological blood disorders and compromised microcirculation in erythrocyte abnormalities $[5,25]$. Effects of the non-neuronal cholinergic elements, especially acetylcholinesterase (AChE), on the erythrocyte hemorheological behaviour were extensively characterized in the past. One of those works 
aimed at assessing the erythrocyte hemorheological behavior under the influence of the cellular redox thiol status [2]. The results of that study showed that the erythrocyte redox thiol status modulates neither the erythrocyte hemorheological parameters (aggregation and deformability) nor the erythrocyte lipid membrane fluidity. The slight changes observed may not reflect any clinical relevance. Nevertheless, in the concomitant presence of acetylcholinesterase substrate (acetylcholine, $\mathrm{ACh}$ ) or inhibitor (velnacrine maleate, VM) several significant alterations were reported, namely a shift on the erythrocyte discoid shape (observed in blood smears) as well as on the aggregation index. The lower aggregation tendency detected may become increasingly important in the clinical setting as far as cellular-activated thiols are concerned. We documented, for the first time in this study, that increasing concentrations of a thiol reducing agent in red cells do not significantly modify the red cell elongation index, aggregation index and membrane lipid fluidity, an effect dependent on the non neuronal AChE modulation.

\subsection{DTT and nitric oxide bioactivity}

In a second study [24], we intended to assess the redox thiol status influence on the nitric oxide (NO) metabolism and efflux in erythrocytes stimulated with ACh and VM. Since sulfhydryl groups are the main nitric oxide receptors into red cells, the $-\mathrm{SH}$ donor function of DTT raised the evidence that the intraglobular redox status, the hallmark of many cell processes, is capable of modulating the release of nitric oxide and its oxidative metabolites which takes place under several physiological conditions (e.g. hypoxia). In this study, upon redox thiol status modulation by DTT, nitric oxide is strongly mobilized inside erythrocytes but much less released to the extracellular compartment, when compared with the erythrocyte suspensions without either DTT or ACh/VM. Increasing DTT concentrations significantly increased the levels of nitrite/nitrate. Concerning the overall NO reactions inside red cells the explanation lies on the NO efflux impairment which is consequently mobilized amongst several nitrosylated compounds and, as a result, higher metabolization into oxidative metabolites occurs. An interesting finding in our study was that reduced glutathione is not modified when DTT or AChE effectors are present, denoting that the cell antioxidant mechanisms are conserved.

This study revealed that nitric oxide is mobilized onto different derivative species, depending upon the erythrocyte-stimulating effector. AChE-triggered enzyme activity alongside redox status shifting seemed to influence the intracellular mobilization of NO-hemoglobin stores. Though still vague, the mechanism of erythrocyte NO mobilization is now proved to be dependent on circulating acetylcholine and on the cellular redox thiol status. Our in vitro model reinforces the thiol redox status and, consequently, NO is less released and more mobilized inside the erythrocyte [24].

\section{Summary}

According to the supracited applications overviewed in this review, dithiothreitol is regarded as the reagent of election as thioreducer agent and is used in a wide range of clinical occurrences. This particularity of reduced status-dependent nitric oxide storage by red cells becomes an important step to comprehend the influence of erythrocyte-dependent microcirculation events and, in a way, to raise new goals as targets upon microcirculation disease. The «endogenous» low-molecular-weight thiols, primarily represented by glutathione and cysteine, are known to participate in a large number of physiological phenomena. Modifications in their basal levels are often on the basis of pathological events, such as imbalance of the intraand extracellular antioxidant/oxidant ratio. Furthermore, some studies reveal thiols may play a central 
role in the pathogenesis of cardiovascular disease and aging. We believe thiol levels measurement may thereby become valuable in the assessment of the antitoxic-antiradical status and to detect risk factors for cardiovascular disease and therapy monitoring.

\author{
Abbreviations \\ AChE, acetylcholinesterase \\ ACh, acetylcholine \\ Cys, cysteine \\ DTT, dithiothreitol \\ NO, nitric oxide \\ $\mathrm{NO}_{2}{ }^{-}$, nitrites \\ $\mathrm{NO}_{3}{ }^{-}$, nitrates \\ $-\mathrm{SH}$, sulfhydryl groups \\ $\mathrm{VM}$, velnacrine maleate
}

\title{
References
}

[1] M.C. Alliegro, Effects of dithiothreitol on protein activity unrelated to thiol-dissulfide exchange: For consideration in the analysis of protein function with cleland's reagent, Analytical Biochemistry 282 (2000), 102-106.

[2] J.P. Almeida, F.A. Carvalho, T. Freitas and C. Saldanha, Modulation of hemorheological parameters by the erythrocyte redox thiol status, Clin Hemorheol Microcirc 40 (2008), 99-111.

[3] J. Antonowicz, R. Andrzejak and T. Lepetow, Influence of heavy metals, especially lead, on lipid metabolism, serum alpha-tocopherol level, total antioxidant status, and erythrocyte redox status of copper smelter workers, Fresenius' J Anal Chem 361 (1998), 365-367.

[4] S.L. Archer, J.A. Will and E.K. Weir, Redox status in control of pulmonary vascular tone, Herz 11 (1986), 127-141.

[5] M. Bláha, E. Rencová, V. Bláha, R. Malý, M. Blazek, J. Studnicka, C. Andrýs, I. Fátorová, S. Filip, M. Kasparová, R. Procházková, J. Malý, R. Zimová and H. Langrová, The importance of rheological parameters in the therapy of microcirculatory disorders, Clin Hemorheol Microcirc 42 (2009), 37-46.

[6] F.A. Carvalho, A.V. Maria, J.M. Braz Nogueira, J. Guerra, J. Martins-Silva and C. Saldanha, The relation between the erythrocyte nitric oxide and hemorheological parameters, Clin Hemorheol Microcirc 35 (2006), 341-347.

[7] F.A. Carvalho, J. Martins-Silva and C. Saldanha, Amperometric measurements of nitric oxide in erythrocytes, Biosens Bioelectron 20 (2004), 505-508.

[8] F.A. Carvalho, J.P. Almeida, I.O. Fernandes, T. Freitas-Santos and C. Saldanha, Non-neuronal cholinergic system and signal transduction pathways mediated by band 3 in red blood cells, Clin Hemorheol Microcirc 40 (2008), 207-227.

[9] F.A. Carvalho, J.P. de Almeida, T. Freitas-Santos and C. Saldanha, Modulation of erythrocyte acetylcholinesterase activity and its association with G protein-band 3 interactions, J Membr Biol 228 (2009), 89-97.

[10] F.A. Carvalho, R. Mesquita, J. Martins-Silva and C. Saldanha, Acetylcholine and choline effects on erythrocyte nitrite and nitrate levels, J Appl Toxicol 24 (2004), 419-427.

[11] W.W. Cleland, Dithiothreitol, a new protective reagent for SH groups, Biochemistry 3 (1963), 480-482.

[12] J.P. de Almeida, T. Freitas-Santos and C. Saldanha, Fibrinogen-dependent signaling in microvascular erythrocyte function: Implications on nitric oxide efflux, J Membr Biol 231 (2009), 47-53.

[13] S. de Oliveira, A.S. Silva-Herdade and C. Saldanha, Modulation of erythrocyte deformability by PKC activity, Clin Hemorheol Microcirc 39 (2008), 363-373.

[14] P. Di Simplicio, M.G. Cacace, L. Lusini, F. Giannerini, D. Giustarini and R. Rossi, Role of protein -SH groups in redox homeostasis - the erythrocyte as a model system, Arch Biochem Biophys 355 (1998), 145-152. 
[15] B.S. Edwards, M.S. Curry, E.A. Southon, A.S. Chong and L.H. Graf Jr, Evidence for a dithiol-activated signalling pathway in natural killer cell avidity regulation of leukocyte function antigen-1: Structural requirements and relationship to phorbol ester and CD16-triggered pathways, Blood 6 (1995), 2288-2301.

[16] M.S. Gelman and J.M. Prives, Arrest of subunit folding and assembly of nicotinic acetycholine receptors in cultured muscle cells by dithiothreitol, J Biol Chem 271 (1996), 10709-10714.

[17] H. Goldman, C.R. Scriver, K. Aaron and L. Pinsky, Use of dithiothreitol to correct cystine storage in cultured cystinotic fibroblasts, Lancet 18 (1970), 811-812.

[18] H. Goko, S. Takashima, A. Kawamuro and A. Matsuoka, Insulin-like effects of dithiothreitol on isolated rat adipocytes, Biochem J 200 (1981), 425-428.

[19] K.D. Held, H.A. Harrop and B.D. Michael, Effects of oxygen and sulphydryl-containing compounds on irradiated transforming DNA. Part I. Actions of dithiothreitol, Int J Radiat Biol Relat Stud Phys Chem Med 40 (1981), 613-622.

[20] B. Hultberg, A. Andersson and A. Isaksson, Interaction of metals and thiols in cell damage and gluthetione distribution: Potentiation of mercury toxicity by dithiothreitol, Toxicology 156 (2000), 93-100.

[21] H. Kleszczynska, D. Bonarska, J. Sarapuk and S. Przestalski, Protection of erythrocytes against organometals-induced hemolysis, J Flouoresc 14 (2004), 5-10.

[22] V.R. Lavis and R.H. Williams, Studies of the insuline-like actions of thiols upon isolated fat cells, J Biol Chem 245 (1970), 23-31.

[23] V.V. Lauriault and P.J. O'Brien, Molecular mechanism for prevention of N-acetyl-p-benzoquinoneimine cytotoxicity by the permeable thiol drugs diethyldithiocarbamate and dithiothreitol, Mol Pharmacol 40 (1991), 125-134.

[24] J.P. Lopes de Almeida, F.A. Carvalho, A.S. Silva-Herdade, T. Santos-Freitas and C. Saldanha, Redox thiol status plays a central role in the mobilization and metabolism of nitric oxide in human red blood cells, Cell Biol Int 33 (2009), 268-275.

[25] A. Marossy, P. Svorc, I. Kron and S. Gresová, Hemorheology and circulation, Clin Hemorheol Microcirc 42 (2009), 239-258.

[26] R. Mesquita, B. Piçarra, C. Saldanha and J. Martins e Silva, Nitric oxide effects on human erythrocytes structural and functional properties - an in vitro study, Clin Hemorheol Microcirc 27 (2002), 137-47.

[27] T. Okuno and N. Kondelis, Evaluation of dithiothreitol (DTT) for inactivation of IgM antibodies, Fresenius' J Anal Chem 361 (1978), 365-367.

[28] M.G. Ribeiro, A.R. Pedrenho and A. Hasson-Voloch, Electrocyte $\left(\mathrm{Na}^{+}, \mathrm{K}^{+}\right)$ATPase inhibition induced by zinc is reverted by dithiothreitol, Int J Biochem Cell Biol 34 (2002), 516-524.

[29] D.M. Rothwarf and H.A. Scheraga, Equilibrium and kinetic constants for the thiol-disulfide interchange reaction between glutathione and dithiothreitol, Proc Natl Acad Sci USA 89 (1992), 7944-7948.

[30] U.T. Ruegg and J. Rudinger, Cleavage of disulfide bonds in proteins, Methods Enzymol 47 (1977), 111.

[31] C. Saldanha, A.S. Silva, S. Gonçalves and J. Martins-Silva, Modulation of erythrocyte hemorheological properties by band 3 phosphorylation and dephosphorylation, Clin Hemorheol Microcirc 36 (2007), 183-194.

[32] C. Saldanha, N.C. Santos and J. Martins-Silva, Fluorescent probes DPH, TMA-DPH and $\mathrm{C}_{17}-\mathrm{HC}$ induce erythrocyte exovesiculation, J Membr Biol 190 (2002), 75-82.

[33] N.C. Santos, C. Saldanha and J. Martins e Silva, A colorimetric process to visualize erythrocyte exovesicles aggregates, Biochem Biophys Acta 32 (2004), 250-253.

[34] N.C. Santos, C. Saldanha and J. Martins e Silva, Gramicidin D and dithiothreitol effects on erythrocyte exovesiculation, Cell Biochem Biophys 43 (2005), 419-430.

[35] T. Santos, R. Mesquita, J. Martins e Silva and C. Saldanha, Effects of choline on hemorheological properties and NO metabolism of human erythrocytes, Clin Hemorheol Microcirc 29 (2003), 41-51.

[36] B.R. Schwartz and J.M. Harlan, Sulfhydryl reducing agents promote neutrophil adherence without increasing surface expression of CD11b/CD18 (Mac-1, Mo1), Biochem Biophys Res Commun 165 (1989), 51-57.

[37] L.B. Tee, A.R. Boobis, A.C. Huggett and D.S. Davies, Reversal of acetaminophen toxicity in isolated hamster hepatocytes by dithiothreitol, Toxicol Appl Pharmacol 83 (1986), 294-314.

[38] H.T. Truong, J.E. Jr Ferrell and W.H. Huestis, Sulphydryl reducing agents and shape regulation in human erythrocytes, Blood 67 (1986), 214-221.

[39] H.T. Truong, D.L. Daleke and W.H. Huestis, Dithiothreitol stimulates the activity of the plasma membrane aminophospholipid translocator, Biochim Biophys Acta 1150 (1993), 57-62.

[40] D. Qiu and W.C. Tan, Dithiothreitol has a dose-response effect on cell surface antigen expression, J Allergy Clin Immunol 103 (1999), 873-876.

[41] M.B. Zucker and N.C. Masiello, Platelet aggregation caused by dithiothreitol, Thromb Haemost 51 (1984), 119-124. 